\title{
PAS DE SORTIE FACILE. IMMENSITÉS, INTIMITÉS ET INTENSITÉS SIBÉRIENNES CHEZ ANDREÏ MAKINE
}

\author{
Diana MisTREANU \\ Université Paris-Est/Université du Luxembourg
}

\begin{abstract}
En): This paper focuses on the image and the role of the chronotope of Soviet Siberia in three of Makine's novels: Le testament français, Au temps du fleuve Amour and L'amour humain. It argues that while the endless landscapes, harsh winters and political prisons which marked Siberia are indeed depicted in these texts, other - and more significant - dimensions are added to it, such as its representation as a land of individual freedom and - thanks to the discovery of French movies and literature on its territory - of intellectual growth. Thus, this paper proposes a complex and non-Manichaean reading of Makine's Siberia, hypothesising that this immense territory ultimately functions as a hypertrophied uterus which symbolically gave birth not only to the writer, but also to the integrality of his literary output.
\end{abstract}

Keywords (En): Siberia; palimpsest-space; anthropomorphic space; lignes de fuite

Mots-clés (Fr): Sibérie ; espace-palimpseste ; anthropomorphisation de l'espace ; lignes de fuite

« [...] au-delà de l'Oural, derrière cette frontière invisible, mais si matérielle de l'Europe » Andreï Makine, Au temps du fleuve Amour

\section{Introduction}

Malgré l'absence d'une étude sur les représentations sociales de la Sibérie dans la culture occidentale, il nous semble que ce toponyme évoque des associations essentiellement dysphoriques, colportées par la littérature et l'iconographie dont il fait l'objet. Aussi dans une carte politique dressée à Liège en 1864 est-il désigné par deux indications : « route de l'exil » et « ténèbres de la Barbarie » ${ }^{1}$. Suivant la même logique, le 12 avril 1865 les lecteurs du Courrier du Grand-Duché de Luxembourg apprennent que «la mortalité est grande en Sibérie à cause des conditions climatériques, hygiéniques et autres, de la misère des habitants, de celle plus grande encore des déportés polonais» $(1865: 3)$. De nos jours, une des publications les plus récentes à ce sujet s'intitule The House of the Dead: Siberian Exile Under the Tsars (BEER, 2017), la Sibérie y étant présentée comme une vaste prison sans toit. C'est donc à un public dont la connaissance de la Sibérie est médiée par de telles sources que s'adressent les romans d'Andreï Makine, le Russe élu en 2016 à l'Académie française. L'auteur propose cependant une image moins réductrice de sa terre natale, dont nous explorerons la représentation dans trois romans, symptomatiques de l'ensemble de son œuvre'2, à savoir Au temps du fleuve Amour

\footnotetext{
1 Carte des États désunis d'Europe, Liège, 1864. Source : http://weburn.kb.se/metadata/540/digkart_10348540.htm.

${ }^{2}$ Elle comprend jusqu'à présent (août 2017) un essai, une pièce de théâtre, quinze romans publiés sous son nom, dont Le Testament français, récompensé par le Prix Goncourt en 1995, quatre romans parus sous le pseudonyme de Gabriel Osmonde, ainsi que ses contributions à l'album photographique de
} 
(1994), Le testament français (1995) et L'amour humain $(2006)^{3}$. Il s'agira, dans les lignes qui suivent, d'identifier les marqueurs textuels de la représentation de la Sibérie et la place de celle-ci dans la trame des ouvrages, pour démontrer qu'elle se décline sous trois aspects : celui de l'immensité géographique, celui de l'intimité dont elle est vecteur et celui de l'anthropomorphisation de l'espace. Ajoutons d'entrée de jeu que par «représentation» nous entendrons désormais, tout simplement, mise en texte.

\section{Immensités ${ }^{4}$}

Cela ne surprendra personne : il existe, de prime abord, une Sibérie placée sous le signe de la démesure géographique et historique. Par exemple, le narrateur du Testament français, un écrivain francophone d'origine soviétique émigré à Paris, relate les souvenirs des étés de son enfance ${ }^{5}$, qu'il passait chez sa grand-mère française, dans une petite ville « aux abords de la steppe russe où [celle-ci] avait échoué après la guerre » (1995: 17). L'isotopie de l'immensité spatiale est d'emblée manifeste. La ville de Saranza - toponyme imaginaire ${ }^{6}$ - est décrite comme étant « figée à la bordure des steppes dans un étonnement profond devant l'infini qui s'ouvrait à ses portes » (1995: 38), et les plaines que l'enfant voit depuis le balcon de l'appartement sont «sans limites» (1995: 32) - expression qui fait écho à «l'infini russe » (1995:23) et à «l'immensité neigeuse de la Russie» (1995: 17) qui ondoie «de la Mer Noire jusqu' au Pacifique » $(1995: 32)$ «sous le soleil des steppes » $(1995: 23)$. Qui plus est, la steppe dont il est question est la grande steppe eurasiatique, la plus vaste formation de ce type au monde, qui commence en Europe de l'Est et finit en Extrême-Orient, couvrant la plus grande partie du sud de la Sibérie. Sa dimension renforce celle que l'URSS avait à l'époque (22,4 millions de $\mathrm{km}^{2}$, dont elle a perdu un peu plus de cinq millions lors de sa dissolution) et explique l'hyperbolisation fréquente de l'espace qui, d'ailleurs, a des frontières floues. En effet, le narrateur sent qu'il vit « au milieu de la steppe » $(1995: 125)$, « au bout du

Ferrante Ferranti sur la ville de Saint-Pétersbourg et à celui d'Elena Maldevskaïa sur le costume populaire russe.

${ }^{3}$ Le nom de l'auteur ne sera plus mentionné dans les citations suivantes extraites de ces trois textes.

${ }^{4}$ C'est l'anthologie de textes russes d'Élisabeth BARILlÉ, Le goût de la Russie (Paris, Gallimard, 2015), répartie en trois chapitres dénommés «Immensités », «Intimités » et «Intensités », qui nous a inspiré la structure tripartite de cet article.

${ }^{5} \mathrm{La}$ traduction anglaise de ce roman récupère dans le titre la référence au chronotope, le rendant plus précis et sans doute plus attrayant pour le public anglophone : Dreams of My Russian Summers (1997), traduit par Geoffrey Strachan.

${ }^{6}$ Makine insère des toponymes imaginaires, comme Saranza et Nerloug (Au temps du fleuve Amour), au sein d'espaces qui ont un équivalent dans le monde réel, brouillant ainsi le pacte mimétique de la représentation du monde. Il en existe des antécédents - pensons au village normand d'Yonville dans Madame Bovary. Puisque ce problème dépasse le cadre de notre article, nous nous contenterons de préciser succinctement ici qu'il nous semble que ce procédé est une mise en abyme de la création littéraire, qui ne consiste pas dans l'imitation du monde (dit) réel, mais dans l'insertion, dans le monde réel et à partir de celui-ci, d'un univers imaginaire, mais possible. Par ailleurs, pour éviter tout malentendu, précisons que Saranza se trouverait non pas en Sibérie, mais dans la partie européenne de la steppe eurasienne, dans la région de la Volga. La Sibérie apparaît dans Le testament français dans les souvenirs de Charlotte. 
monde, [dans] ce désert sibérien » (1995: 74), et dans ce contexte, la recherche et la perte des repères spatiaux est à mettre en relation avec sa recherche de repères émotionnels, intellectuels et spirituels. Avide de connaissances et en quête de soi, Aliocha passe ses étés « russes » à lire l'histoire et la littérature françaises, et de ce fait, il (se) construit et déconstruit constamment des identités problématiques ( $c f$. SYLWESTRZAK-WSZELAKI, 2010), tantôt russes, tantôt françaises, qui voleront finalement en éclat pour laisser leur place à une identité paratopique ${ }^{7}$, celle d'écrivain. Or, prise dans le vertige de ce processus, la steppe joue un rôle crucial, puisqu'elle participe à la genèse du chronotope matriciel de l'enfance du narrateur - mais nous y reviendrons. Contentons-nous de retenir, pour l'instant, que nous pouvons appeler ce texte "le roman de la steppe».

Mutatis mutandis, Au temps du fleuve Amour est « le roman de la taïga », car l'adolescence des trois protagonistes, Outkine et Samouraï et le narrateur, Mitia, se passe dans la taïga de la région du fleuve Amour, dans l'Extrême-Orient russe. À l'instar de la steppe, la taïga, constituée par des forêts de conifères, est une formation vaste qui couvre presque entièrement la partie centrale et septentrionale de la Sibérie, ainsi que la péninsule Scandinave. L'immensité sibérienne et ses contours brouillés apparaissent dans ce texte notamment à travers deux éléments. Le premier est la réitération de l'adjectif infini, déterminant du calme de la taïga (1994: 28 et 36), alors que le second réside dans l'isotopie de la sensation d'éloignement qu'éprouvent les protagonistes, dont la vie aux confins de l'empire façonne leur représentation du monde et relativise les notions d'Orient et d'Occident. Les héros passent leur «jeunesse enfouie au fin fond de la Sibérie orientale » (1994:16), dans une « contrée austère » (1994:17) où ils fantasment sur la vie qui existe «à dix-mille kilomètres de ces lieux, par-delà l'Oural, à l'autre bout de l'empire » (1994:33) - en Ukraine ou dans le Caucase. Les distances et les frontières sont donc remises en cause. La ville de Novossibirsk, qui est la plus grande ville sibérienne, leur semble « presque aussi irréelle que la Crimée » (1995: 39), car leur Europe, qu' ils nomment simplement « Occident », commence au bord du Baïkal (1994 : 39) - ce qui déplace la frontière réelle du continent de $3435 \mathrm{~km}$ (la distance qui sépare Ekaterinbourg d'Irkoutsk).

À l'immensité de l'espace correspond la démesure climatique, exprimée par les marqueurs textuels de l'intensité des phénomènes météorologiques, signes d'une nature indomptable. Ainsi, dans Au temps du fleuve Amour, le climat corrobore l'immensité de la terre et intensifie la sensation d'emprisonnement : «l'Asie nous tient par ses espaces infinis, par l'éternité de ses hivers » (1994 : 189-190). En effet, si l'adjectif «vaste » est, selon Bachelard, un des mots les plus baudelairiens (1957: 174), « infini » est sans aucun doute un des mots les plus makiniens. Dans la citation ci-dessus, il correspond, en chiasme, à « l'éternité », la syntaxe reflétant la manière dont le topos et le chronos se complètent l'un l'autre. «Les grands froids » (1994: 60) y sont également mentionnés, de même que l'«abondance neigeuse » et les

\footnotetext{
${ }^{7}$ Selon Dominique MAINGUENEAU, la paratopie est la condition par excellence pour devenir créateur. «Élaborer sa paratopie, c'est ainsi découvrir cette modalité singulière de ne-pas-trouver-sa-place qui permet de faire œuvre. [Elle est] à la fois ce qui rend l'œuvre possible et ce que cette œuvre configure et légitime en lui donnant sens. » (2004: 6-7)
} 
« immenses dunes de neige» (1994: 94) apportées par les vents du Pacifique (1994 : 29) dans « le pays des neiges éternelles » (1994:14).

De même, la première chose qu'Elias Almeida - le révolutionnaire professionnel angolais qui est le héros de L'amour humain - remarque lorsqu'il arrive en Sibérie avec son amie, Anna, est l'impact somatique du « froid extrême [qui] noircit la peau plus que la brûlure du soleil » (1994 : 157). Plus tard, en route vers Sarma, le village natal d'Anna, situé à l'est du Baïkal ${ }^{8}$, ils sont sur le point de geler et ils sont retrouvés par un chauffeur local qui « aurait gardé le même calme si, sous l'abri, il avait retrouvé leurs corps figés» (1994: 163). Le calme du chauffeur transmet - avec une remarquable économie langagière - un aspect fondamental de la vie de tous les jours des riverains, à savoir le fait que périr gelé n'a rien de choquant pour un Sibérien.

«L'immensité blanche » (1995: 70) réapparaît dans Le testament français, lorsque Charlotte décrit la Sibérie comme une suite de «lieux sauvages » dont la terre est gelée et les bourrasques de neige, fréquentes (1995 : 74). En outre, la neige contribue aussi à l'amplification de la sensation de perte des repères spatiaux, dans un pays où la nature confère à l'espace une attirance fatale. C'est au moins de cette manière que le narrateur explique que son arrière-grand-mère Albertine - dont le prénom est un clin d'œil à Proust - ait préféré la Sibérie à Paris après la mort de son mari : « la planète des neiges ne relâchait jamais les âmes envoûtées par ses espaces sans jalons, par son temps endormi » $(1995: 70)$, idée reprise quelques paragraphes plus loin, où la Sibérie devient «absurde et inévitable comme le destin » (1995: 73). La métaphore cosmique «planète des neiges » indique la quantité et l'étendue de la neige en renforçant son omniprésence, car, comme Nina Nazarova le constate pertinemment, « la neige est partout dans les livres de Makine : un village enneigé succède à l'autre et ses personnages, ensevelis par la neige, semblent tourner à pas de valse dans ses orages » $(2005: 115)$. Pour Nazarova, la fréquence de ces images est due à la nostalgie de la neige, dont souffriraient les Russes émigrés en Occident.

Par ailleurs, la neige symbolise aussi l'attente, la résignation et la pureté, elle a le pouvoir d'agir sur l'espace et de le remodeler, d'en cacher les apparences et d'y ajouter d'autres dimensions. Selon Gilles Lapouge :

La neige efface la géographie. Ou plutôt, elle ajoute à la géographie une autre géographie, quelque chose comme son contraire, un cache, un transparent. Elle adoucit les aspérités et elle lisse les lointains. Quand elle est très lourde, elle enlève les chemins. Alors on ne sait plus par quel bout attraper le paysage. Elle le découpe, elle rassemble les pièces du puzzle et elle les colle ensemble. Elle en masque les jointures. Le temps glisse sur ses miroirs. La neige maltraite. Elle le pile. Elle en fait de petits bouts d'éternité. Elle cache les choses qui existent. D'autres choses, qui n'existent pas, pointent le nez. La neige tombe dans l'envers du monde. (LAPOUGE, 2009: 177)

Effectivement, chez Makine la neige efface les chemins, recouvre les villages, bloque les personnages dans leurs isbas, uniformise le paysage et lui ajoute une dimension opalescente et diaphane. Considérée dans cette perspective, elle fait

\footnotetext{
${ }^{8}$ À la différence de Nerloug et de Saranza, le village de Sarma se trouve bien à l'endroit indiqué dans le texte, à savoir au bord du Baïkal.
} 
pendant à l'État, puisqu'à l'instar de celui-ci, elle s'empare de l'espace pour le transformer. Elle est aussi la preuve ultime qu'il existe des forces qui dépassent l'humain et le politique.

La démesure caractérise en égale mesure l'histoire de la Sibérie. Ainsi, cet espace porte les stigmates de son passé récent, celles du célèbre Goulag (acronyme de l'Administration principale des camps, en russe, Главное управление лагерей) créé par Staline en 1934 pour centraliser la gestion des camps, et qui a compté 18 millions de prisonniers, dont 4,5 millions n'en revinrent jamais (APPELBAUM, 2005 : quatrième de couverture $)^{9}$. Chez Makine, cette question n'occupe le devant de la scène que dans son plus récent roman, L'archipel d'une autre vie (2016), alors que dans les autres, les camps sont évoqués de manière récurrente, mais en filigrane. Ils apparaissent ainsi dans les souvenirs des vieux et dans l'imaginaire des jeunes. Par exemple, le narrateur de L'amour humain se souvient que dans son enfance, « de très vieux hommes assis près de leurs isbas [...] regardaient au loin et parlaient d'êtres qui ne vivaient plus que dans leurs têtes blanches, lourdes des années de guerre et de camps » (2006 : 18-19). Dans un autre passage du même roman, quand Elias et Anna sortent se promener dans la neige, ils rejoignent les ruines d'un camp où «les mots perdaient [...] leur sens. Sur un poteau noirci pendait un bout de rail dont le goulag cadençait autrefois l'activité du camp. Désormais sa mutité ressemblait à une présence invisible mais toujours vivante» (2006: 173). On observe ainsi que chez Makine, la violence du passé traverse le temps et l'espace pour imprégner l'intégralité du territoire sibérien, ce qui crée un effet d'omniprésence de la souffrance, renforcé par la façon dont Anna décrit son lieu natal à Elias : «il n'y avait que ça dans notre village, des prisonniers sortis des camps » (2006: 139). L'histoire, cependant, reste inénarrable. Elle est évoquée succinctement, car «l'histoire cruelle de cet immense empire, de ses famines, révolutions, guerres civiles...» (1995: 102) ébranle la logique de l'existence.

Quoi qu'il en soit, il nous semble que s'il existe un aspect digne de notre attention dans la représentation de la Sibérie, celui-ci ne découle certainement pas de la mise de sa géographie et de son histoire sous le signe de la démesure. En effet, la Sibérie recouvre environ $77 \%$ de la Russie actuelle, elle s'étend sur plus de 13 millions de $\mathrm{km}^{2}$ - soit presque $10 \%$ de la surface terrestre de notre planète -, la plus grande partie de son territoire a un climat sous-polaire avec des hivers froids où la température descend jusqu'à $-60^{\circ} \mathrm{C}$ et elle a été utilisée comme prison politique depuis le XVII ${ }^{\mathrm{e}}$ siècle, avec l'institution de la katorga dans la Russie tsariste (le camp de travail forcé). Qui plus est, au $\mathrm{XX}^{\mathrm{e}}$ siècle elle a hébergé la plupart des plus de 30000 camps du Goulag. Dans cette logique, la démesure reste, certes, la toile de fond, incontournable d'ailleurs, de la Sibérie makinienne, mais sur elle se greffe un espace présenté sous un autre jour, qui déplace le centre d'intérêt vers une zone beaucoup plus délicate: celle des rencontres avec autrui, de la construction identitaire et du devenir.

\footnotetext{
${ }^{9}$ Tous les historiens ne sont pas d'accord avec ces chiffres. Pour une problématisation du nombre des détenus du Goulag, voir APPELBAuM (2005 : 629-635).
} 


\section{Intimités}

La Sibérie peut aussi rendre libre, protéger et favoriser les échanges intimes et participer à l'épanouissement spirituel et intellectuel. Cela est moins paradoxal qu'il ne le paraît, puisqu'une image euphorique de la Sibérie existe déjà dans la littérature et l'histoire russes, particulièrement au XIX ${ }^{\mathrm{e}}$ siècle, quand, comme Mark Bassin le remarque, « some romantic writers discovered that in Siberia's unspoiled landscape they could regain a pure, inspirational bond with the organic natural world, a bond long lost in civilized and despiritualized European Russia » (1991 : 782-783). Polevoi, Ivan Kalashnikov ou Nikolai Shchukine en ont ainsi laissé des descriptions valorisantes. Il en va de même pour certains décembristes - officiers de la garde impériale qui ont organisé une révolte contre le nouveau tsar Nikolaï ${ }^{\mathrm{er}}$ en décembre 1825 et ont été condamnés à l'exil - car, contrairement à leurs attentes, les membres de cette élite intellectuelle ont trouvé en Sibérie une terre fertile à cultiver et un endroit propice pour propager leurs idées progressistes ( $c f$. SUTHERLAND, 1984 et BARRATT, 1974).

De la même manière, les protagonistes de Makine s'y sentent protégés et libres. Par exemple, Elias y échappe à la discrimination raciale qu'il subit à Moscou, où il avait été agressé par une bande de jeunes qui l'avaient frappé et traité de « primate » et de « macaque » (2006: 134), alors que les « confins de l'empire, lieu d'échouage des vies malmenées, des êtres indésirables dans les métropoles [...] brassait une multitude d'ethnies et de coutumes, univers bigarré qui accueillait cet Africain comme une nuance de plus dans le chaos de la mosaïque humaine » (2006 : 158). Ces mêmes confins de l'empire abritent ses échanges romantiques - et platoniques - avec Anna, leurs promenades dans la neige et les moments où ils épanchent leurs cœurs, partagent leurs craintes et leurs désirs et se rapprochent l'un de l'autre avec tendresse. Accueillant ainsi dans son giron les individus fatigués et malmenés, la Sibérie participe à la construction du double binôme antithétique capitale/province et Russie/Asie, qui traverse l'œuvre makinienne. Profondément asiatique, colonisée par les Russes, elle s'oppose cependant à tout ce qui peut être considéré comme moscovite, à savoir le poids de l'aspect politique de la vie, l'intolérance et l'architecture stalinienne, monolithique. Ainsi, affranchissant les personnages des contraintes propres aux métropoles, la steppe et la taïga sibériennes deviennent propices à l'amour romantique et à l'intimité des âmes et des esprits. L'univers intime se dévoile lorsqu'il est confronté à l'immensité extérieure.

En ce qui concerne le rôle de la Sibérie dans la formation intellectuelle et spirituelle, il réside dans deux éléments. Le premier est la nature, qui favorise aussi l'introspection et la méditation car, comme le souligne Yvon le Caro, au contraire des espaces clos, les espaces ouverts nourrissent la tolérance et privilégient les retours sur soi-même, la paix et la solitude (2007). Aussi la steppe fait-elle partie du chronotope matriciel dans Le testament français - dans l'acception où Bakhtine entend ce concept, à savoir celle de «matérialisation de temps dans l'espace » autour duquel gravitent «tous les éléments abstraits du roman, généralisations philosophiques et sociales, idées, analyse des causes et des effets » (1978: 391). Le rôle qu'elle joue ici doit être mis en relation avec ses connotations dans la culture russe, liées au rôle pluriséculaire qu'elle a joué dans l'élaboration du mythe de la 
conquête et de l'exploration de l'inconnu. Pour citer Daria Sinichkina, « la steppe n'a cessé d'exciter l'imagination des explorateurs, poètes et écrivains. De $L a$ Steppe (1888) de Tchékhov à celle de Tchinguiz Aïtmatov, cet espace est l'incarnation paradoxale du plus exotique et du plus familier, véritable tableau vivant du caractère humain autant qu'exutoire de ses craintes les plus intimes » (2012). Or, ce qu'Aliocha retient des étés de son enfance sont justement les après-midis qu'il passait sur le balcon de sa grand-mère, suspendu « au-dessus de la steppe sans fin » (1995 : 170), d'où il contemplait le paysage, écoutait les récits de Charlotte et lisait des romans français. Le balcon, situé symboliquement au bord de la structure végétale, représente le passage d'un monde à l'autre et fait écho aux oscillations d'Aliocha entre la France lointaine et fantasmée et sa Russie de tous les jours, fascinante mais incompréhensible. Il est aussi le seuil visible entre l'espace clos et habité de la maison et la steppe illimitée et sauvage. Incarnant la frontière entre nature et culture, il permet au héros à la fois de se livrer à son intimité et de s'ouvrir vers un extérieur inconnu et indéfini, qui est le prix à payer pour accéder à un nouvel horizon. Chose intéressante, sa description renvoie à un objet magique, propre aux contes merveilleux : «Le balcon tanguait légèrement, se dérobant sous nos pieds, se mettant à planer. L'horizon se rapprocha comme si nous nous élancions vers lui à travers le souffle de la nuit» (1995 : 28). En vérité, les verbes employés ici «tanguer», « planer » et «élancer »- semblent désigner non pas une construction immobile, mais un tapis volant, qui est propre à la mythologie perse et arabe, mais qui existe également dans le folklore russe - on peut le voir, par exemple, dans les célèbres tableaux de Viktor Vasnetsov inspirés des contes traditionnels ${ }^{10}$. Associé au voyage, au dédoublement et à l'ubiquité, le tapis volant représente un moyen symbolique de parcourir le monde, or c'est exactement dans ce but qu'Aliocha utilise le balcon de sa grand-mère. Qui plus est, l'association entre balcon et tapis volant devient explicite dans un autre passage, où le narrateur mentionne le « balcon volant» (1995: 29) suspendu «au-dessus de la steppe sans fin » (1995: 170) qui rend possible l'exploration d'espaces chimériques et lointains. «Rabelais [...] hantait nos soirées de steppe » (1995: 121, nous soulignons), dit le narrateur, et à côté de lui il y avait Proust, Flaubert, José-Maria de Heredia et Molière. La configuration de l'espace et la proximité de la nature engendrent et entretiennent les fantasmes de l'adolescence et favorisent la lecture et la rêverie.

Le deuxième élément qui façonne la vie des personnages consiste dans les objets culturels étrangers qui pénètrent l'espace sibérien, à savoir la littérature, déjà évoquée ci-dessus, la cinématographie et la gastronomie françaises. Il nous semble que les propos de Ian Buruma et Avishai Margalit peuvent éclairer la raison du « succès » de ces produits parmi les jeunes sibériens. Selon les deux chercheurs, la globalisation est une source de séduction infinie dans les déserts culturels (2016 : 48), et la vie des personnages makiniens se déroule souvent dans des endroits en proie à une pénurie culturelle, comme, par exemple, la région du fleuve Amour du roman éponyme. Originaires d'un village, les protagonistes de ce livre se rendent

\footnotetext{
${ }^{10}$ Il apparait notamment dans deux huiles sur toile réalisées en 1880 et en 1926, intitulées toutes les deux Le tapis volant.
} 
régulièrement au cinéma Octobre rouge de la petite ville de Nerloug ${ }^{11}$ pour regarder les films où joue Jean-Paul Belmondo. Pour les trois adolescents, l'acteur devient un mythe - dans le sens où Barthes emploie ce terme dans Mythologies, c'est-à-dire un système de représentations collectives (1957: 7). Aussi parcourent-ils de nombreux kilomètres sous la neige pour revoir un de ses films dix-sept fois, et ils apprennent ses répliques par cœur. Certes, l'absence de diversité culturelle des villages sibériens n'est pas la seule explication du succès de Belmondo, puisque d'une part, comme le remarque Jean-Michel Guy, parmi les personnalités nationales, ce sont les personnalités françaises qui sont les mieux connues à l'étranger (2008 : 14), et de l'autre, l'Union soviétique héritait déjà d'une longue tradition francophone - en dépit de la relégation de la francophilie au statut de marque de l'élitisme culturel impérial. Mais par-delà les dimensions historique et sociologique du phénomène, l'essentiel réside dans la portée mythique de l'acteur, qui actualise un imaginaire convoité et bouleversant :

L'arrivée de Belmondo avait interrompu le cours régulier du temps. L'hiver avait perdu sa signification de sommeil éternel. Les soirées - à cause des films - celle d'apaisement vespéral. L'instant de dix-huit heures trente s'était imposé à tous avec une évidence cosmique. Nous vivions au gré de ces rythmes nouveaux, nous retrouvant aujourd'hui au Mexique, demain à Venise. Toute autre temporalité était caduque... (1994:227)

Dans ce passage, la description de «l'arrivée de Belmondo» renvoie directement à l'isotopie du cycle des saisons (l'arrivée du printemps, l'arrivée des cigognes etc.). Elle marque un autre rapport au temps et inaugure un nouveau rythme de vie, où la culture finit par l'emporter sur la nature : «Impossible de me souvenir si c'était l'An I ou l'An II de notre nouvelle chronologie » (1994 : 227, nous soulignons), dira le narrateur plus loin. Décidément, Belmondo subvertit la démesure climatique et politique, mettant le hic et nunc entre parenthèses pour placer les personnages sous le signe de l'ailleurs et du possible. Comme l'observe pertinemment Elisabetta Abignente, le savoir-faire ironique, l'apparence et les manières séduisantes du personnage représenté par l'acteur français produisent un effet de rupture dans la vie quotidienne et l'imagination des jeunes, dont il devient l'unique modèle et héros $(2011: 3)$.

En outre, le vocabulaire culinaire français produit le même effet, en l'occurrence, sur l'esprit du petit Aliocha et de sa sœur dans Le testament français. Lorsqu'ils sont en train de faire la queue pour la nourriture et qu'ils apprennent qu'il n'y en a pas assez pour tout le monde, ils se remémorent des noms de plats français - dont le référent réel leur est, bien entendu, inconnu - qui acquièrent la valeur d'une formule magique :

Malgré le cri d'une vendeuse [...], les gens continuaient à affluer. [...] Nous restions au bout de la file, hypnotisés par la puissance anonyme de la foule. [...] Et c'est comme venant d'une autre planète que j'entendis soudain la voix de ma sœur - quelques paroles teintées d'une mélancolie souriante :

- Te rappelles-tu : Bartavelles et ortolans truffés rôtis ?... [...]

\footnotetext{
11 Voir note 5.
} 
Et moi, en regardant son visage pâle aux yeux qui reflétaient le ciel d'hiver, je sentis mes poumons s'emplir d'un air tout neuf - celui de Cherbourg - à l'odeur de la brume salée, des galets humides sur la plage, et des cris sonores des mouettes dans l'infini de l'océan. Je restai un moment aveugle. La file d'attente avançait et me poussait lentement vers la porte. Je me laissai faire sans quitter cet instant de lumière qui se dilatait en moi. (1995:68-69)

L'accès à l'univers gastronomique français n'efface pas les différences de classe, mais il permet de se détacher du présent et offre une autre vision du monde :

Nous appartenions tous à la même classe : celle des gens qui pataugeaient dans une neige piétinée au milieu d'une grande ville industrielle, aux portes d'un magasin, en espérant remplir leurs sacs de deux kilos d'oranges. Et pourtant, en entendant les mots magiques appris au banquet de Cherbourg, je me sentis différent d'eux. Non pas à cause de mon érudition (je ne savais pas, à l'époque, à quoi ressemblaient les fameux bartavelles et ortolans). Tout simplement, l'instant qui était en moi - avec ses lumières brumeuses et ses odeurs marines - avait rendu relatif tout ce qui nous entourait : cette ville et sa carrure très stalinienne, cette attente nerveuse et la violence obtuse de la foule. (1995: 69)

Peu à peu, les frontières mêmes de l'Union soviétique, qui s'ouvraient avec difficulté pour les petites gens, sont transgressées par un imaginaire actualisé tantôt par un livre, tantôt par un film ou par le nom d'un plat. Face à la littérature, à la cinématographie et à la gastronomie françaises, le présent vole en éclat.

Les éléments qui rendent ce processus possible, et sur lesquels il convient de nous arrêter un instant, correspondent à ce que Gilles Deleuze et Félix Guattari appellent des lignes de fuite ${ }^{12}$, qui font partie des trois types de lignes identifiées par les deux philosophes, à côté des lignes dures et des lignes souples. Les lignes dures appartiennent aux dispositifs du pouvoir et concernent notre formation au sein des institutions de l'État, notre carrière et notre parcours, depuis la scolarité jusqu'à la retraite. Les lignes souples, quant à elles, nous permettent de nous détacher de l'égide de l'organisation sociale, mais sans la déstabiliser. Elles consistent dans nos désirs cachés, nos conversations privées, nos cours séchés et nos petites révoltes de tous les jours, à la suite desquels nous reviendrons sur les lignes dures. Les lignes de fuite, en revanche, remettent profondément en question notre rapport avec l'organisation sociale et politique, puisqu'elles opèrent des ruptures irrémissibles. À la différence d'une ligne souple, une ligne de fuite est réelle, et elle est vectrice d'émancipation et de liberté. Lorsque je suis sur une ligne de fuite, disent les auteurs, «plus personne ne peut rien pour moi ni contre moi. Mes territoires sont hors de prise, et pas parce qu'ils sont imaginaires, au contraire, parce que je suis en train de les tracer » $(1980: 244)$.

La culture française confère justement cette intangibilité aux héros. Comme nous venons de le voir, face au monde que les vocables français actualisent dans l'imaginaire du narrateur, l'architecture stalinienne, la faim et le comportement bestial de la foule perdent leur sens - et mentionner Staline dans ce contexte ne fait

\footnotetext{
${ }^{12}$ Pour une analyse de l'œuvre de Makine à travers les théories de Deleuze et Guattari, consulter la thèse de doctorat d'Erzsébeth Harmath (Université de Szeged), publiée à Paris en 2016 et disponible en ligne depuis 2011. Nous remercions d'ailleurs l'auteure et sa directrice, Madame le Professeur Timea Gyimesi, car notre réflexion sur les lignes de fuite chez Makine s'appuie directement sur leur recherche.
} 
que renforcer le pouvoir des mots. Le narrateur lui-même en est conscient, car il confesse, lors d'une réflexion rétrospective sur son enfance, que porter en soi ces références «n'était pas innocent. [...] c'était bel et bien un défi, une provocation aux yeux de ceux qui vivaient au présent » (1995 : 154-155). Il en va de même pour les protagonistes d'Au temps du fleuve Amour. Jean-Paul Belmondo leur offre une identité nouvelle et laisse une trace indélébile sur leurs existences, à tel point que « rien ne sera plus jamais comme avant » (1994 : 226). En effet,

[s]on grand portrait à côté de L'Octobre rouge disparut, cédant la place à quelques visages maussades d'un film sur la guerre civile. Mais l'Occident était là, parmi nous. On sentait sa présence dans l'air du printemps, dans la transparence du vent dont nous percevions parfois le goût piquant, océanique, dans l'expression détendue des visages. (1994: 220)

Ces passages montrent que la présence de la culture française en Sibérie a un pouvoir déstabilisateur, ne serait-ce que parce qu' elle entraîne la prise de conscience de l'individualité au sein d'un monde qui promeut le collectivisme. Elle trace des territoires autres, qui se trouvent non seulement par-delà les frontières nationales, mais surtout dans l'esprit des héros, dans leur capacité d'imaginer, de rêver et, finalement, de créer.

\section{Intensités}

La Sibérie est inextricablement liée aux moments de rupture biographique, inoubliables et indélébiles, qui marquent le passage à un autre cycle de vie ou la proximité de la mort, et elle est surtout présente dans les contextes amoureux. En effet, l'association entre nature sibérienne et amour est récurrente chez Makine, son corollaire étant l'anthropomorphisation de l'espace. Il s'agit de la troisième image de l'Asie russe, qui concerne la féminisation de la Sibérie, déclinée sous deux aspects : la Sibérie-amante et la Sibérie-mère.

Mais avant toute chose, il convient de nous arrêter sur la conception makinienne de l'amour. Nous partageons à cet égard l'avis de Nina Nazarova, selon qui amour et érotisme «paraissent y mener une existence parallèle, sans jamais se croiser. L'amour, pour Makine, est un sentiment sublime tandis que l'érotisme remonte à la nature bestiale de l'homme » - retenons la modulation («paraissent »), car amour et érotisme peuvent en fait se croiser chez Makine. Nazarova remarque aussi, et à juste titre, que lorsque l'auteur «parle de l'amour comme d'un sentiment élevant l'homme, il a en vue surtout la génération de ses parents et de ses grands-parents » (2008: 92). Si l'amour est donc lié à une sorte de passéisme, ce n'est pas son occurrence qui est impossible dans le présent de la narration, mais son accomplissement, et cela est dû au fait que la femme cède aux pressions sociales et matérielles, et elle quitte l'homme aimé pour se marier avec un membre du Parti et assurer ainsi son avenir. Ce scénario narratif est récurrent - on le retrouve dans $L a$ musique d'une vie (2001), L'amour humain (2006), Le livre des brèves amours éternelles (2011) et L'archipel d'une autre vie (2016), et il n'est pas impossible qu'il soit d'inspiration autobiographique.

Toutefois, si amour et érotisme ne se confondent pas au niveau conceptuel, ils se superposent au niveau lexical, dans le langage et l'imaginaire des personnages. 
Ainsi, l'amour romantique est dans un premier temps associé à la France, tandis que l'érotisme vulgaire et déshumanisant est associé à la Russie. Dans Le testament français, la source de cette perception de «l'amour français » réside dans la littérature et les récits de Charlotte, qui déterminent Aliocha à se demander si la quintessence française ne serait pas l'amour - la France y est appelée « le pays du Tendre ». Cet amour, tel que décrit ici, reste néanmoins romanesque, à l'image des «frasques de la divine Otero » et de Casque d'Or (1995 : 123), où la mort en duel ou le suicide sont une preuve de dignité et de grandeur d'âme. Un des symboles de cet «amour français » est le couple Félix Faure - Marguerite Steinheil, et la disparition du Président à « l'Élysée, dans les bras de sa maitresse » (1995: 112), renforce le binôme manichéen France-Russie :

Secrétaires du Politburo, maîtres du Kremlin: Lénine, Staline, Khrouchtchev, Brejnev. Quatre caractères fort différents aimés ou détestés par la population, et dont chacun avait marqué toute une époque dans l'histoire de l'empire. Pourtant, ils avaient une qualité en commun : à leur côté, aucune présence féminine et, à plus forte raison, amoureuse n'était concevable. Il était bien plus facile pour nous d'imaginer Staline en compagnie d'un Churchill à Ialta ou d'un Mao à Moscou que de le supposer avec la mère de ses enfants... (1995: 112)

Décrire les relations entre hommes et femmes en URSS exige donc l'emploi d'un langage différent, «d'une obscénité monstrueuse » (1995: 113), capable de rendre le caractère bestial de l'érotisme russe.

C'est dans ce contexte que s'inscrit la première hypostase de l'espace anthropomorphe, où les frontières géographiques et politiques sont de nouveau remises en cause car - chose étrange - la steppe eurasiatique est associée non pas à l'amour russe, mais à l'amour français et à la femme maîtresse par excellence. On apprend ainsi que lorsque Charlotte contait les aventures d'Otero, "Saranza plongeait dans la nuit épicée des steppes. Ses senteurs se confondaient avec le parfum qui embaumait ce corps féminin couvert de pierreries et d'hermine » (1995: 121-122). Cette projection d'attributs féminins sur la steppe déconstruit le binôme France-amour romantique/Russie-érotisme bestial construit auparavant, car elle confère à la nature russe un caractère séduisant et sensuel, essentiellement féminin, donc « français ». Le phénomène devient moins paradoxal si on le considère comme un travail de compensation opéré par la psyché du narrateur, qui attribue à la nature - surface de projection de ses désirs et rêves intimes - ce dont la culture et l'imaginaire politique le privent.

Le même type de compensation est à l'œuvre dans Au temps du fleuve Amour, lorsque Mitia confronte sa conception de l'amour charnel, inspirée par les films de Belmondo, à son expérience personnelle, qui s'avère frustrante et décevante. Avant son initiation érotique avec une prostituée, Mitia croyait que "l'amour aurait l'intensité de notre plongeon nocturne dans la neige, Samouraï et moi, sous le ciel glacé. Cet instant unique où le feu du bain et le froid des étoiles donnaient naissance à une fusion fulgurante » (1994:75). La réalité s'inscrit cependant en faux contre sa vision cosmique de l'union physique : «La matière de l'amour était donc telle : glissante, visqueuse... Et les amants, pesants, essoufflés. C'était comme si chacun, péniblement, tirait le corps de l'autre... Mais où ? [...] Je ne savais pas si ce que j'éprouvais était peur, pitié, amour ou dégoût » (1994: 76). La confusion et le 
désenchantement changent le narrateur profondément, à tel point qu'avec sa vision initiale de l'amour, c'est également son identité qui éclate :

Si je ne me jetai pas tout de suite dans l'abîme blanc, c'est qu'en arrivant au sommet du pont je constatai qu'il n'y avait plus de moi. Il n'y avait plus personne à précipiter vers la rive gelée. [...] Je me retrouvai sur le pont avec quelques loques de moi qui se dispersaient dans l'obscurité fouettée par la neige. (1994:81)

Les métaphores de l'abîme blanc et du sommet renvoient à l'union des corps, dont le narrateur souhaite en vain récupérer la dimension sublime. Par ailleurs, Aliocha et Mitia ont recours au même mécanisme : ils projettent sur quelque chose d'autre (la France et la nature sibérienne respectivement) ce dont la vie réelle les prive. La taïga enneigée et la steppe deviennent des surfaces de projection de désirs romanesques, de rêves intimes, de l'amour raté et, en définitive, dans la mesure où elles symbolisent la page blanche à remplir, de la vocation d'écrivain, existante en germe chez les deux personnages.

Le testament français et Au temps du fleuve Amour sont des romans de formation qui portent sur la fin de l'enfance et l'adolescence, l'initiation, la découverte et le désir intellectuel et amoureux. Cela explique cette représentation de la Sibérie en tant que femme aimée, avec les glissements, les projections et les transferts que les mécanismes des conflits intrapsychiques inhérents à l'âge des héros mettent en œuvre. L'amour humain, en revanche, est la rétrospective d'une vie faite à l'âge adulte par Elias Almeida, et la Sibérie n'y est pas séduisante, mais maternelle. Son premier attribut concerne la dimension protectrice de la mère, transférée sur un endroit particulier qui devient l'abri ultime, synonyme de sollicitude et d'acception. Il apparaît dès l'incipit du roman, quand, Elias, croyant qu'il mourrait bientôt, demande au narrateur de retenir une adresse qui sera « le seul refuge sûr, le lieu où l'on revient après avoir tout perdu et où l'on est certain d'être accepté tel qu'on est » (2006 : 20). Ce lieu est l'isba des parents d'Anna. La dénomination périphrastique de cet endroit fait écho à l'évocation par Elias de sa propre mère lorsqu'il se souvient d'un épisode de son enfance où, effrayé par les guerres sanglantes, il «s'accroupit et cacha son visage là où ce monde embrouillé n'existait pas, dans le creux chaud et tendre du coude de sa mère » $(2006: 48)$. Le coude maternel est source de vie, mais également d'amour et de vérité : «La vie y coulait, sommeilleuse, bercée par le battement du sang, une vie tout autre, sans le rictus des morts sur les routes, sans mensonge » (2006 : 48). Elias ressent la même chose quand la mère d'Anna l'invite à prendre un bain, expérience qui ressemble à une résurrection du corps, donc à une nouvelle naissance :

Tout était étonnant pour lui dans cette minuscule isba des bains : l'odeur amère des murs enfumés, les rameaux de bouleau avec lesquelles il était censé se fouetter, la vapeur qui brûlait les narines. Derrière la vitre floue, le froid interdisant toute ombre de vie et là, sur les planches inondées d'eau bouillante, son corps nu, plus vivant que jamais. (2006:165)

Il est intéressant de confronter la description du creux du coude maternel à celle du bain, car ces deux passages portent sur la seconde dimension de la Sibérie-mère, à savoir celle de la mère génitrice. Éloignés l'un de l'autre et dans l'économie du 
livre et dans la chronologie du récit, ils sont tous les deux construits sur une double opposition, entre l'intérieur et l'extérieur et entre la vie et la mort. L'intérieur du coude, comme celui de l'isba, correspond à la vie. Dans « [1]a vie y coulait, bercée par le battement du sang, une vie tout autre », le pronom adverbial désigne l'espace qui est pour Elias-enfant vecteur de vie, en contraste avec celui qui se trouve en dehors du coude de la mère, parsemé de cadavres et de mensonges. De même, Eliasadulte perçoit le contraste entre le monde funeste situé « derrière la vitre floue », qui avait banni « toute ombre de vie », et celui de l'intérieur de l'isba, où il se sent «plus vivant que jamais». Le participe passé adjectivisé «bercée » renvoie aux bercements de l'enfance, alors que l'isba remplie d'eau chaude, avec le corps nu d'Elias à l'intérieur de ses murs, recrée le ventre maternel, avec son liquide amniotique - l'eau matricielle dans laquelle baigne le fotus.

L'anthropomorphisation de la Sibérie, qui est tantôt la femme aimée, tantôt une mère aimante, renforce la «fertilité » de l'usage littéraire de cet espace, qui revêt une multitude de masques. Immense, imprévisible, vecteur de violence et de chagrins, elle s'avère aussi, comme nous venons de le voir, intime et protectrice. Elle déclenche et inspire la création littéraire et offre un décor propice pour cultiver son esprit. Elle accueille les humiliés et donne des leçons aux innocents. Finalement, la dynamique des interactions entre ses trois représentations - immense et effrayante, intime et instructive, séduisante et maternelle - reproduit celle, tout aussi inextricable, qui régit les rapports entre les personnages et l'espace.

\section{Conclusions}

La Sibérie est représentée chez Makine à travers le triple prisme de sa démesure, souvent dangereuse et anxiogène, de sa capacité à protéger les personnages et à favoriser leur épanouissement intellectuel et leurs échanges intimes, et, enfin, de son anthropomorphisme. Sa démesure, évoquée dans la première partie de notre article, est inhérente à sa géographie et fonctionne comme une toile de fond pour les deux autres dimensions sibériennes. Son fonctionnement en tant que catalyseur de l'évolution intellectuelle et affective des personnages la transforme en une ligne de fuite - dans l'acception deleuzo-guattarienne de cette notion -, rendant possible la dissociation des personnages de l'époque historique où ils vivent par l'actualisation d'un imaginaire européen qui devient une source de rêverie et de liberté spirituelle. Si cette deuxième dimension entraîne l'exil, qui apparaît d'abord en tant que possibilité, avant de se matérialiser, ce sont les hypostases féminines de cet espace qui font en sorte que les narrateurs exilés y retournent mentalement pour interroger les topoï essentiels de l'existence humaine : l'amour, la mort et le sens de la vie. La Sibérie est donc, comme nous venons de le voir, un palimpseste à trois couches qui s'inscrit en faux contre toute interprétation manichéenne. Ce n'est ni un locus hostilis par excellence - bien que l'auteur ne se dérobe pas à l'évocation des atrocités commises sur son territoire, ni un locus amoenus par définition, en dépit de son association au paradis perdu de l'enfance. Elle porte en elle ces dimensions, mais aussi bien d'autres. À peine le lecteur apprend-il qu'elle est oppressive, fatale et impossible à quitter lorsqu'il est informé que le narrateur se trouve non pas dans l'Extrême-Orient russe, mais à Paris ou à New-York, et que c'est dans ces grandes 
villes occidentales qu'il se remémore sa vie sibérienne. Ainsi, en dépit de nos efforts, nous craignons que tout essai de classification du palimpseste sibérien, quelque méticuleux qu'il se veuille, soit voué à l'échec parce que contaminé de cet inénarrable qui est pour l'écrivain à la fois le privilège et la malédiction des choses importantes, des aspects cruciaux de la vie humaine. «Discourir sur les futilités est facile, mais qui trouvera la langue qui puisse dire l'Amour, le Beau, le Vrai, en bref, l'essentiel ? » - voilà la question qui sous-tend la totalité des textes de cet auteur. Une chose nous semble, toutefois, certaine : la Sibérie est la terre natale de l'écrivain et une des sources primordiales de son imaginaire. Elle est l'endroit où naissent et vivent ses héros et l'espace matriciel de son œuvre. Les personnages peuvent la quitter, mais elle ne les quittera jamais. À la lumière de ces considérations, une question nous hante : la Sibérie - et particulièrement la Sibérie soviétique - seraitelle un ventre hypertrophié, un utérus gigantesque qui a donné naissance non seulement à Andreï Makine, mais aussi à l'intégralité de son œuvre ?

\section{BIBLIOGRAPHIE}

\section{Corpus}

MAKINE Andreï (1994), Au temps du fleuve Amour, Paris, Gallimard.

MAKINE Andreï (1995), Le testament français, Paris, Mercure de France.

MAKINE Andreï (2006), L'amour humain, Paris, Seuil.

\section{Ouvrages cités}

ABIGNENTE Elisabetta (2011), Il limite e l'altrove: il mito di Jean-Paul Belmondo nella Siberia di Andreï Makine. Between, I.1, p. 1-15, http://www/Betweenjournal.it/.

APPlEBAUM Anne (2005), Goulag : une histoire, Paris, Grasset.

BAKHTINE Mikhaïl (1978), Esthétique et théorie du roman, Paris, Gallimard.

BARILLÉ Élisabeth. (2015), Le goût de la Russie, Paris, Gallimard.

BARrATT Glynn (1974), Voices in Exile, Montreal, McGill-Queen's University Press.

BARTHES Roland (1957), Mythologies, Paris, Seuil.

BEER Daniel (2017), The House of the Dead: Siberian Exile Under the Tsars, New York, Knopf.

Buruma Ian; MARGAlit Avishai (2016), Occidentalismul. Războiul împotriva Occidentului: o scurtă istorie a urii faţă de Vest, Bucureşti, Humanitas.

Deleuze Gilles; GuATTARI Félix (1980), Capitalisme et schizophrénie. Mille plateaux, Paris, Minuit.

GUY Jean-Michel (2008), Cultures croisées. Références interculturelles des Allemands, des Italiens et des Français, Culture études, 6, p. 1-32.

HARMATH Erzsébet (2016), Andreï Makine et la francophonie. Pour une géopoétique des ouvres littéraires, Paris, L'Harmattan.

LAPOUGE Gilles (2009), La légende de la géographie, Paris, Albin Michel.

LE CARO Yvon (2007), Les loisirs en espace agricole. L'expérience d'un espace partagé, Rennes, Presses universitaires de Rennes. 
MAINGUENEAU Dominique (2004), Le discours littéraire : paratopie et scène d'énonciation, Paris, Armand Colin.

NaZArova Nina (2005), Andreï Makine. Deux facettes de son ouvre, Paris, L'Harmattan.

S.A. (1865), Autriche, Courrier du Grand-Duché de Luxembourg, 12/4.

SINICHKINA Daria (2012), L'enfant des steppes dans l'imagerie et la littérature soviétique pour enfants (1917-1953), Strence, 3, http://strenae.revues.org/568 ; DOI : $10.4000 /$ strenae.568.

Sutherland Christine (1984), The Princess of Siberia: The Story of Maria Volkonsky and the Decembrist Exiles, New York, Farrar, Straus and Giroux.

SYLWESTRZAK-SZELAKI Agata (2010), Andrë̈ Makine. L'identité problématique, Paris, L'Harmattan. 